\title{
Annette Karmiloff-Smith (1938-2016): Precursora de una psicología del desarrollo interdisciplinaria ${ }^{12}$
}

\author{
David Garnica Agudelo \\ Magister en Neurociencias \\ Göttingen Graduate Center for Neurosciences, Biophysics, \\ and Molecular Biosciences (GGNB), Alemania \\ Clinical Neurophysiology, University Hospital Göttingen, Alemania \\ Universidad El Bosque, Bogotá, Colombia \\ Correo electrónico: david.garnica@med.uni-goettingen.de
}

Ana Milena Franco Rueda

Magister en Psicología Clínica Corporación Universitaria Minuto de Dios, Bogotá, Colombia Correo electrónico: anmfrancoru@unal.edu.co
Recibido: 23/01/2019

Evaluado: $25 / 02 / 2019$

Aceptado: 26/03/2019

\section{Resumen}

Con motivo del fallecimiento de Annette Karmiloff-Smith, en este artículo se presenta una revisión de la obra y trayectoria investigativa de esta autora, haciendo especial énfasis en sus aportes a la psicología del desarrollo, la neuropsicología y la ciencia cognitiva contemporáneas. Se presentan los elementos centrales de su propuesta teórica y las principales evidencias que dan sustento empírico a su perspectiva sobre el desarrollo cognitivo típico y atípico. Adicionalmente, se rastrea el impacto de sus ideas en las discusiones actuales en psicología y ciencia cognitiva, y se explican algunas líneas de trabajo que la autora misma planteó para la investigación futura. En general, la obra de KarmiloffSmith resulta relevante en la investigación actual en psicología debido a las líneas de investigación específicas pero comunes que abordó entre la psicología, neurociencia y genética, planteando un antecedente colaborativo y metodológico entre estas áreas que seguirá ofreciendo evidencias sobre los factores que caracterizan la variabilidad del desarrollo neurocognitivo humano.

Palabras clave

Neuroconstructivismo, desarrollo cognitivo, psicología del desarrollo, neuropsicología, neurociencia.

12 ara citar este artículo: Garnica, D. \& Franco, A.M. (2020). Annette Karmiloff-Smith (1938-2016): Precursora de una psicología del desarrollo interdisciplinaria. Informes Psicológicos, 20(1), pp. 183-200 http://dx.doi.org/10.18566/ infpsic.v20n1a12 


\title{
Annette Karmiloff-Smith (1938- 2016): Precursor of an interdisciplinary developmental psychology
}

\begin{abstract}
On the occasion of the death of Annette Karmiloff-Smith, this article presents a review of the work and research career of this author, with special emphasis on her contributions to contemporary developmental psychology, neuropsychology and cognitive science. The central elements of her theoretical proposal and the main evidence that give empirical support to her perspective on typical and atypical cognitive development are presented. Additionally, the impact of her ideas on current discussions in psychology and cognitive science is traced, and some lines of work that the author herself proposed for future research are explained. In general, Karmiloff-Smith's work is relevant in current research in psychology due to the specific but common lines of research she addressed between psychology, neuroscience and genetics, posing a collaborative and methodological background between these areas that will continue to offer evidence on the factors that characterize the variability of human neurocognitive development.
\end{abstract}

Keywords

Neuroconstructivism, cognitive development, developmental psychology, neuropsychology, neuroscience.

\section{Annette Karmiloff-Smith (1938-2016): Precursora de uma psicologia interdisciplinar do desenvolvimento}

\section{Resumo}

Por ocasião da morte de Annette Karmiloff-Smith, este artigo apresenta uma revisão da trajetória, trabalho e pesquisa desta autora, com ênfase especial em suas contribuições para a psicologia do desenvolvimento, neuropsicologia e ciência cognitiva contemporânea. São apresentados os elementos centrais de sua proposta teórica e as principais evidências que dão suporte empírico à sua perspectiva sobre 0 desenvolvimento cognitivo típico e atípico. Além disso, é traçado o impacto de suas ideias nas discussões atuais em psicologia e ciência cognitiva, e são explicadas algumas linhas de trabalho que a própria autora propôs para pesquisas futuras. Em geral, o trabalho de Karmiloff-Smith é relevante nas pesquisas atuais em psicologia devido às linhas de pesquisa específicas, mas comuns, que ela abordou entre psicologia, neurociência e genética, apresentando um fundo colaborativo e metodológico entre essas áreas que continuarão a oferecer evidências sobre os fatores que caracterizam a variabilidade do desenvolvimento neurocognitivo humano.

Palavras chave Neuroconstrutivismo, desenvolvimento cognitivo, psicologia do desenvolvimento, neuropsicologia, neurociência. 


\section{ntroducción}

Annette Karmiloff-Smith nació en Londres, Inglaterra el 18 de Julio de 1938. Sus padres, Isaac (sastre) y Doris Smith (administradora) le inscribieron en la escuela gramática de Edmonton County, donde estudió hasta 1949 para luego terminar estudios básicos en el Instituto Francés de Londres en 1957. Su gusto por las lenguas la llevó a trabajar como traductora de Naciones Unidas en Ginebra, Suiza. Al parecer esto no llenó sus expectativas intelectuales $\mathrm{y}$, al atender una de las conferencias de Jean Piaget, decidió estudiar psicología experimental. Terminó sus estudios de licenciatura en la Universidad de Ginebra en 1970 y sus estudios de doctorado en 1977. Fue parte del Centro Internacional de Epistemología Genética, en el que permaneció hasta 1980, y trabajó sobre el desarrollo cognitivo normal en varias áreas bajo la tutela de Jean Piaget y Bärbel Inhelder (Elman \& Tyler, 2017).

A continuación, laboró de manera periódica en el Instituto Max Planck de Psicolingüística en Nijmegen, Países Bajos. Fue a partir de esta experiencia que inició su trabajo en el desarrollo del lenguaje. En 1982 volvió a Londres para continuar investigando el desarrollo lingüístico normal en la Unidad de Desarrollo Cognitivo MRC de la Universidad de Cambridge. Posteriormente, habiendo creado la Unidad de Desarrollo Neurocognitivo en el Instituto de Salud Infantil de University College London en 1998, se interesó en la investigación de las dificultades cognitivas de los trastornos del neurodesarrollo de base genética. Su trabajo continuó en relación a estos temas de manera interdisciplinaria, colaborando con diferentes grupos de investigación en genética en el Reino Unido. Desde el año 2006 trabajó en la Universidad de Londres, en el Laboratorio de Desarrollo Neurocognitivo en Birkbeck. Hasta su muerte, ocurrida el 19 de Diciembre de 2016, a los 78 años, investigó de manera productiva con un equipo de profesionales y estudiantes de diferentes disciplinas, siempre con un interés profundo en las variables que modulan, constriñen y posibilitan el desarrollo cognitivo (Association for Psychological Science, 2015; 2016; Thomas, 2017; Elman \& Tyler, 2017).

El presente artículo se desarrolla en un orden cronológico, de acuerdo al transcurso temático y metodológico que tuvo la autora a lo largo de su obra. En primer lugar, se explica el inicio de su trabajo, enfocado en el desarrollo temprano del lenguaje y la distancia que marcó de la teoría de Piaget. Posteriormente, se aborda su investigación del desarrollo cognitivo como un proceso de especialización, planteando críticas a los postulados de la modularidad y la representación. En tercer lugar, se expone su acercamiento al conexionismo para plantear una visión interdisciplinaria del desarrollo cognitivo, aportando también al planteamiento del neuroconstructivismo como teoría del desarrollo. Por último, se abordan sus investigaciones sobre las características de trastornos genéticos del desarrollo y el papel que ejercen variables sociales específicas en las diferencias encontradas entre trastornos del desarrollo. 
Investigaciones sobre el desarrollo del lenguaje: Más allá de la postura piagetiana

Fue con la llegada a la década de los 80 que Karmiloff-Smith comenzó a trabajar sobre el desarrollo típico del conocimiento lexical y morfosintáctico de los niños (Karmiloff-Smith, 1979; 1985), proponiendo las bases teóricas de sus argumentos frente a las posturas de modularidad, innatismo y hacia el modelo de neuropsicología del adulto que fueron predominantes para la época. En su libro "A Functional Approach to Child Language: A Study of Determiners and Reference" (Karmiloff-Smith, 1979), la autora asume una doble postura: por una parte, presenta una defensa del constructivismo piagetiano en la explicación del desarrollo del lenguaje y del pensamiento, y de otro lado, critica y toma distancia frente a la postura de su mentor.

La perspectiva de Karmiloff-Smith frente al desarrollo del lenguaje, y que es extensiva a su forma de comprender el desarrollo cognitivo en general, parte de la matriz de la epistemología piagetiana en tanto que coloca en un lugar central la actividad del sujeto como gestor permanente de representaciones a partir de la interacción dinámica con el medio externo físico y socio-cultural, pero también en la recreación continua de las representaciones previamente elaboradas (Tolchinsky, 2017).

Recordemos que para Piaget el desarrollo del conocimiento es comparable al desarrollo biológico: un agente activo tiene una evolución hacia el equilibrio, entendiendo éste último como el logro de la sistematización del conocimiento, en un orden que inicia con esquemas de acción sensorio-motora, continúa con el aprendizaje de símbolos y la adquisición de estructuras operativas con representaciones verbales iniciales, prosigue con la construcción de operaciones lógicas pero concretas y termina con la aparición de operaciones hipotéticas y abstractas que permiten hacer generalizaciones y proposiciones verificables (Köhler, 2008; Piaget \& Inhelder, 1969/2000; Piaget, 1964/1991). Por otra parte, el modelo de cambio cognitivo de Piaget supone la presencia de un conjunto de esquemas (estructura) que deben incluir conocimiento nuevo (asimilación), cambiar para incluir información suficiente (acomodación) y permitir al sujeto comprender la mayoría de situaciones sin ajustes adicionales (equilibración y adaptación) (Köhler, 2008; Piaget, 1964/1991).

Específicamente, la perspectiva de Karmiloff-Smith mantiene relación con la postura piagetiana en aspectos como la noción de continuidad biológica y la centralidad de la aproximación ontogenética para la comprensión del funcionamiento cognitivo. No obstante, Karmiloff-Smith se distancia de su mentor al proponer otro modelo del cambio cognitivo, la redescripción representacional, por la que el sujeto reelabora las representaciones iniciales sin que esto implique que se siga un orden lineal estricto entre representaciones, estructuras cognitivas y estilos de resolución (la representación verbal no es siempre e inevitablemente de nivel más alto que la sensorio-motriz, pues hay conocimientos cuya representación inicial puede ser verbal) (Tolchinsky, 1996).

En lo que respecta a la adquisición del lenguaje, Karmiloff-Smith plantea que ésta no puede explicarse solamente a partir de las características de la inteligencia sensorio-motriz, tal y como se propone 
desde la perspectiva piagetiana. Desde el punto de vista de Piaget, en principio los niños solo llevan a cabo formas de conducta azarosas y desorganizadas cuyo único propósito es satisfacer las necesidades de cuidado y alimentación básicos para favorecer la supervivencia. Al mismo tiempo, los niños realizan respuestas motoras repetitivas y progresivamente coordinan el conocimiento motor con el sensorial para la formación de esquemas, los cuales dan lugar a un pensamiento representacional. Sobre estos aspectos, Piaget afirmó:

La inteligencia sensorio-motriz, que puede estudiarse entre el nacimiento y la edad de 1.5 a 2 años, es decir, en los comienzos del lenguaje, se distribuye según 6 estadios sucesivos caracterizados por esquemas de acción que se coordinan los unos con los otros según un proceso de autorregulación sumamente regular con correcciones, refuerzos, etc., y únicamente en el sexto estadio - es decircuando la asimilación de los objetos a los esquemas de acción se hace susceptible de ser completada por una asimilación de los objetos entre sí, esto es, por una representación se inicia el lenguaje, y, en este sexto estadio, dichos inicios del lenguaje se benefician de toda una construcción que se ha ido formando anteriormente (Piaget, 1923/1983, p. 184).

Entonces, frente a esta formulación, Karmiloff-Smith plantea su crítica a Piaget partiendo de la observación de una capacidad de respuesta básica de los infantes en etapa sensorio-motriz, entendida como un cambio en el foco atencional ante la presentación de diferentes y repetidos sonidos del habla (fonemas) (Karmiloff-Smith, 1979; 1985). Posteriormente, investigadores como Werker \&
Polka (1993) y Kuhl (2004), hicieron más específica esta observación al encontrar evidencia de un reconocimiento temprano de sonidos diferentes del habla que, incluso para los infantes entre los 8 y 10 meses de edad, se restringe a los fonemas de la lengua materna. En síntesis, Karmiloff-Smith (1979; 1985) anticipó que los infantes probablemente presentan un reconocimiento básico del lenguaje hablado, como un proceso paralelo pero diferente a las acciones sensoriomotrices piagetianas, sin que esta observación implicara un acercamiento a la propuesta de un conocimiento innato para el reconocimiento de fonemas o la adquisición de estructuras gramaticales.

Los planteamientos de KarmiloffSmith parten de la postura piagetiana en sus premisas fundamentales, pero van más allá en tanto permiten dar cuenta de los niveles intermedios de cambio representacional que no siguen trayectorias lineales, que no necesariamente se reflejan de manera directa en lo conductual y que suponen retos para la investigación al incorporar las implicaciones de la interacción entre los diferentes niveles de descripción de un fenómeno (Campos, 2017).

\section{Críticas a la modularidad y el desarrollo como proceso de especialización}

En sus trabajos de la década de los 90, Karmiloff-Smith reafirmó que la postura nativista o innatista no da cabida al papel explicativo del aprendizaje y de la interacción con el ambiente que la adquisición del lenguaje requiere, planteando que conceptos como el de modularidad tampoco dan cuenta del papel del tiempo en el desarrollo. Es así como, en su 
libro "Beyond Modularity: A Developmental Perspective on Cognitive Science" (Karmiloff-Smith, 1992), planteó una postura conciliadora entre el nativismo y el constructivismo. Según ésta, múltiples habilidades de dominio-general tienen una naturaleza innata al permitir una organización temprana de conocimientos (reconocimiento de sonidos, imitación de movimientos o reconocimiento de las formas de las caras), pero estas habilidades presentan cambios a medida que el niño interactúa con el ambiente, se siguen cambios representacionales y se desarrollan habilidades de dominio-específico.

A esta integración entre la existencia de módulos innatos y su naturaleza dinámica le llamó redescripción representacional, concepto con el que buscó explicar el cambio de una inteligencia implícita y no verbal a una inteligencia simbólica y verbal, dando un papel activo al sujeto en el desarrollo cognitivo porque construye un conocimiento teórico y explícito de las características de lo aprendido: el lenguaje, las leyes físicas y el mundo social. En otras palabras, el desarrollo consiste no solo en aprender acciones, posibilitadas por módulos estáticos, sino en construir un conocimiento verbal de las capacidades de las acciones, lo cual inscribe, afecta y dinamiza múltiples dominios cognitivos.

El planteamiento anterior difiere de la postura piagetiana, porque señala que el cambio cognitivo ocurre con una estabilidad en el desempeño y no necesariamente en un estado de desequilibrio, y también es diferente de la modularidad de Fodor (1983) porque el conocimiento adquirido no se restringe a las posibilidades de módulos específicos sino que permite el desarrollo de módulos cada vez más especializados y fomentados por el contexto social.

Esta posición crítica frente a la modularidad se extendió a lo largo de su obra. Propuso una modularidad relativa, concepto que sostiene que el cerebro en desarrollo no se encuentra con módulos localizados o especializados al nacer sino que gradualmente los adquiere a lo largo de un tiempo ontogenético (KarmiloffSmith, 1998a; D'Souza \& Karmiloff-Smith, 2011). De esta forma, la modularidad como arquitectura cognitiva es un producto final del tiempo del desarrollo y no el punto de partida por prescripciones innatas (genéticas estrictas).

Karmiloff-Smith expuso diferentes argumentos para esta aproximación a partir de las investigaciones sobre el procesamiento facial y el desarrollo del lenguaje, entre los cuales se encuentran: 1) El reconocimiento facial en infantes de 6 meses de edad presenta activación cerebral bilateral, pero durante la segunda mitad del primer año el hemisferio derecho progresivamente domina esta función (Johnson, 2005); 2) El cerebro adulto también

1 Según Fodor (1983) la arquitectura mental se constituye por una serie de módulos que presentan las siguientes características: 1) Especificidad de dominio, son mecanismos altamente específicos con una función relativamente circunscrita y un campo de aplicación; 2) Aplicación obligatoria, responden automáticamente a los estímulos de la clase apropiada, en lugar de estar bajo un control cognitivo; 3) Rapidez, transforman un input en un output lo bastante rápido para ser empleado en el control en tiempo real de la acción; 4) Encapsulación de la información, el procesamiento modular no se ve afectado por lo que ocurre en otras partes de la mente o arquitectura mental; 5) Arquitectura neural fija, frecuentemente es posible identificar determinadas regiones del cerebro asociadas con tipos particulares de procesamiento; y 6) Patrones de alteración específicos, el procesamiento modular puede fallar o comprometerse de maneras altamente determinadas (estas alteraciones dan pistas sobre la forma y estructura de ese procesamiento). 
presenta aprendizaje y procesos de especialización, una evidencia es que el área fusiforme facial (AFF) se activa con el progresivo entrenamiento para el reconocimiento de objetos artificiales (Gauthier, Skudlarski, Gore, \& Anderson, 2000)2; 3) La mayoría de niños con hemisferectomía izquierda realizada en edad temprana posteriormente tienen puntajes normales en pruebas de lenguaje y atienden escuelas regulares, desempeñándose significativamente mejor que los adultos con daño en el hemisferio izquierdo y áreas del lenguaje (Stiles, Bates, Thal, Trauner \& ReiIly, 2002; Liégeois, Connelly, Baldeweg \& Vargha-Khadem, 2008)3; y 4) La explicación de por qué los niños con Trastorno Específico del Lenguaje (TEL) tienen un desempeño muy pobre en tareas no-verbales pero bueno en pruebas de lenguaje no es necesariamente que presentan de manera innata un módulo no-verbal comprometido, sino que uno o más déficits de bajo-nivel en la infancia temprana pueden afectar en grados variables el lenguaje en un tiempo crítico, planteando una probabilidades para diferentes trayectorias de desarrollo lingüístico (Bishop, 2011; Moyle, Stokes \& Klee, 2011; Choudhury \& Benasich, 2011) ${ }^{4}$.

Adicionalmente, una noción de modularidad cerebral pierde su poder explicativo y resulta ser relativa porque las investigaciones recientes en neurociencia cognitiva apuntan a que el procesamiento de múltiples modalidades sensoriales ocurre en paralelo a lo largo de distintas y numerosas regiones de la corteza cerebral, el procesamiento de la información sensorial está modulada por mecanismos de retroalimentación directa y no directa (forward-backward feedback), y que diferentes áreas cerebrales se activan al realizar una tarea (D'Souza \& Karmiloff-Smith, 2011). Entre algunas de las evidencias respectivas está el hecho de que con una actividad como la lectura de labios sin estímulo auditivo se activan la corteza primaria auditiva y áreas auditivas circundantes (Stein \& Stanford, 2008; Pekkola et al., 2005), que el procesamiento de información auditiva en la corteza auditiva está modulada por proyecciones provenientes de la corteza visual y del surco temporal superior (Besle et al., 2008) y que durante el reconocimiento de rostros la activación de la AFF se da junto a la de áreas como la amígdala, áreas extraestriada y temporal medial de la corteza visual (V5/MT) (Van de Riet, Grezes \& de Gelder, 2009).

\section{Hacia un enfoque interdisciplinario del desarrollo \\ Por otra parte, un enfoque multidisci- plinario para el estudio del desarrollo fue}

2 Esto sugiere que la AFF presenta una activación para el reconocimiento de rostros en los adultos, no porque la región esté pre-especificada para esto, sino debido a sus propiedades relevantes para la experticia visual como habilidad más amplia y que se han especializado a lo largo de la amplia experiencia del individuo con esa clase de estímulos (D'Souza \& Karmiloff-Smith, 2011, p. 279).

3 A partir de esto la autora defendía que los procesos de plasticidad cerebral en la niñez son, entre otros, claves para entender que la modularidad es el resultado del desarrollo, pues la lesión cerebral adulta afecta los módulos ya localizados y especializados, mientras que la lesión cerebral infantil abre otras posibilidades de trayectorias de desarrollo típico o atípico.

4 Cualquier déficit temprano (congénito o adquirido) en habilidades de menor nivel como la atención compartida, comprensión de intenciones comunicativas, segmentación y detección de patrones en la corriente del habla o discriminación fonética y fonémica, puede alterar los procesos de mayor nivel del desarrollo del lenguaje: comprensión y producción (Bishop, 2011; Moyle, Stokes \& Klee, 2011). 
evidente ya en la coautoría de KarmiloffSmith del libro "Rethinking Innateness: A Connectionist Perspective on Development" (Elman et al., 1996). Junto a Jeffrey Elman, Elizabeth Bates y Mark Johnson, la autora defendió un marco conceptual y metodológico para el estudio del desarrollo humano que integra el conexionismo, la teoría de sistemas dinámicos y la neurociencia básica. Al ser las redes neuronales artificiales un modelo del funcionamiento de las redes neuronales biológicas y la teoría de sistemas dinámicos un marco que busca explicar el cambio cognitivo, se tiene que los hallazgos de las investigaciones en neurociencia básica y con modelos conexionistas son una forma de entender cómo se da el cambio cognitivo y así los mecanismos de desarrollo. En este sentido, Elman et al. (1996) no solo proponen una línea de investigación sino también una forma de investigar los mecanismos por los que el conocimiento y el comportamiento se desarrollan a partir de constricciones tanto ambientales como innatas.

Correspondientemente, Elman et al. (1996) defienden, como mecanismo de desarrollo, la existencia de constricciones innatas en relación a la interacción entre la experiencia y los mecanismos neurobiológicos: constricciones de la arquitectura cerebral que subyacen a las formas de conocimiento (p.e., tipo y funcionamiento de las neuronas, su organización en áreas particulares del cerebro e interconexiones entre las áreas) y constricciones cronotópicas (los eventos y sus tiempos en el desarrollo del cerebro que hacen posibles o imposibles ciertos tipos de comportamientos en edades diferentes o con factores ambientales distintos; p.e., la mielinización axonal o la poda sináptica).
De esta forma, los planteamientos de Karmiloff-Smith sobre una modularización progresiva y relativa y de un estudio multidisciplinario del desarrollo, permitieron a otros autores asociados a ella proponer el concepto de especialización interactiva dentro de un marco teórico llamado neuroconstructivismo (Mareschal et al., 2007; Westermann et al., 2007). Esta teoría rechaza el reduccionismo materialista y la independencia de los niveles de descripción de David Marr (1982) al asumir que el estudio objetivo de las trayectorias de desarrollo debe tener consistencia al inter-relacionar los niveles genético, celular, cerebral, corporal y social.

La investigación del desarrollo cognitivo y cerebral, entonces, debe asumir que la cognición está constreñida por los niveles de descripción por debajo de la misma. En otras palabras, el neuroconstructivismo plantea que la cognición y el comportamiento se desarrollan de acuerdo a la interacción multidireccional (top-down y bottom-up) de las distintas variables que conforman los niveles de análisis (desde el genético hasta el corporal y social). Así, por ejemplo, las regiones cerebrales adquieren funciones a lo largo del desarrollo dependiendo de su base genética (incluyendo mutaciones), la estructura y el funcionamiento de sus células, la actividad integrada entre ellas y la pro-actividad del sujeto mismo (exploración y actividad con el medio). Las implicaciones de esta propuesta son abarcadoras, pues plantea el desarrollo como un proceso de especialización biológica y cognitiva, dando cabida a la probabilidad de que ocurran constricciones en cualquier nivel de análisis, y en cualquier tiempo, que conduzcan a diversas trayectorias de desarrollo típico o atípico [de esto trata la epigénesis probabilística 
de Gottlieb, (2007)]. ${ }^{5}$ Al mismo tiempo, el neuroconstructivismo plantea que este proceso de especialización no solo presenta constricciones para el desarrollo de habilidades de dominio general o específico, sino que también hay factores genéticos o ambientales que pueden hacer que un dominio cognitivo facilite la especialización de otro (Mareschal et al., 2007).

A partir de las bases teóricas y metodológicas anteriores, Karmiloff-Smith continuó su investigación empírica abordando varios desordenes del desarrollo, en particular aquellos con una base genética. Este trabajó incluyó desórdenes como el Trastorno del Espectro Autista (TEA), Síndrome de Down (SD), Síndrome de Williams (SW), Síndrome de X frágil (SX), Síndrome velocardiofacial (SVCF) y trastornos del aprendizaje como la discalculia. Se destacan los estudios sobre dificultades en el reconocimiento de rostros de niños con TEA, SD y SW (Annaz et al., 2009; Dimitriou et al., 2015); el pobre desempeño en tareas de conciencia fonológica y vocabulario como predictores longitudinales de desarrollo atípico de la lectura de niños con SD y SW (Steele et al., 2013); la deficiencia en la discriminación de conjuntos pequeños y grandes de objetos (numerosidad) de prescolares con SW (Van Herwegen et al., 2008); la correlación entre deleciones parciales del gen LIMK1 y la cognición espacial en pacientes con SW (Gray et al., 2006; Smith et al., 2009); la correlación entre diferencias morfológicas de la materia gris y blanca cerebral con dificultades atencionales y menor $\mathrm{Cl}$ no-verbal de niños con SW (Campbell et al., 2009a; Campbell et al., 2009b); la relación indirecta entre la base genética del SD con el desarrollo de enfermedad de Alzheimer (EA); y dificultades cognitivas y sociales de niños con SVCF (Stevens et al., 2005).

El abordaje de Karmiloff-Smith sobre el desarrollo atípico ejemplifica su apuesta por la complejidad en la comprensión del desarrollo (D'Souza, D'Souza, \& Karmiloff-Smith, 2017) e impulsa un camino alternativo de investigación que da particular importancia a las asociaciones entre síndromes y a la superación del análisis específico de las alteraciones más evidentes (Karmiloff-Smith, 1998b). El interés se desplaza entonces hacia la comprensión de los procesos y las trayectorias de desarrollo que permiten identificar cómo cambios sutiles en el estado inicial pueden desencadenar diferencias de dominio específico en los estados finales y cómo "en la génesis de funcionamientos posteriores puede haber mecanismos ontogenéticamente más tempranos, de más bajo nivel, que además pueden ser morfológica y funcionalmente muy diferentes a los procesos que aparecerán alterados posteriormente" (Campos, 2018, p. 127).

5 Incluyendo a Karmiloff-Smith, el énfasis dado a las trayectorias de desarrollo por parte de los proponentes del neuroconstructivismo fue la principal razón para que dieran una amplia importancia al diseño longitudinal para el estudio del desarrollo, considerando el diseño transversal como uno que ofrece solo una "vista estática" del desarrollo. Sin embargo, esta teoría ha encontrado varios críticos. Por ejemplo, Ramus (2004) que defiende que el diseño transversal describe el estado del sistema cognitivo en un tiempo dado, buscando abarcar y correlacionar múltiples variables a ese estado, y Farina (2016) que sostiene que posible que la organización estructural y funcional del cerebro cambie en cualquier momento del ciclo vital dependiendo de las prácticas y actividades promovidas por la cultura. 
El dominio social en la propuesta neuroconstructivista

La propuesta del neuroconstructivismo recoge los planteamientos de Rogoff (1990; 1998; 2003) sobre la condición del niño situado, a lo largo de las diferentes etapas del desarrollo, no solo en un mundo físico sino también en un contexto social. El desarrollo cognitivo es entonces un acto colaborativo entre las acciones del niño y las de los agentes sociales que retroalimentan el desarrollo. En consecuencia, Karmiloff-Smith y Mareschal et al. (2007) sostienen que las acciones sociales de los demás también tienen un papel importante en la emergencia del conocimiento (representaciones), es decir, el conocimiento es construido a partir de las condiciones de una base neurobiológica pero también de acuerdo a las posibilidades y condiciones de enseñanza de los actores sociales ("ensocialment").

Consideraciones como éstas se reflejaron en la opción de Karmiloff-Smith et al. (2012) de trabajar sobre las características diferenciales o semejantes entre desórdenes del desarrollo, ampliando la indagación a los efectos probables que pueden tener las distintas condiciones sociales sobre las trayectorias de desarrollo atípico y típico. Por ejemplo, el TEA y el SW son genéticamente distintos ${ }^{6}$ y fenotípicamente parecen tener perfiles sociales bien diferentes: mientras que los individuos con TEA prefieren conservar distancia y muestran desconcierto al ver a otros a los ojos y su rostro, los individuos con SW son desinhibidos y se fascinan por los ojos y rostros de los demás.
En este sentido, pueden compararse los grados de compromiso de habilidades sociales entre distintos desórdenes del desarrollo y explorar correlaciones neurobiológicas respectivas.

Al mismo tiempo, Karmiloff-Smith et al. (2012) resaltan que quedan muchas incógnitas por resolver alrededor de los síndromes neurogenéticos. A diferencia de los niños de ambientes de estado socioeconómico bajo, muchos niños con estos desórdenes crecen en ambientes de considerable cuidado, reciben mayor estimulación cognitiva y presentan menor riesgo de sufrir abusos físicos y/o mentales. Preguntan entonces, ¿por qué realmente los ambientes positivos no compensan las constricciones o vulnerabilidades genéticas, a pesar de que en otros niveles sí se observa la dinámica de compensación?, ¿es posible que las diferencias sutiles entre ambientes tempranos de los individuos tengan efectos variables sobre el desarrollo?

La postura del neuroconstructivismo plantea constricciones genéticas y condiciones socio-ambientales variables en magnitud y tiempo que pueden dar lugar a trayectorias de desarrollo con distintas probabilidades. En este sentido, la presencia de un desorden del neurodesarrollo no solo implica mutaciones genéticas sino también la presencia de variables que modifican el ambiente en el que el infante se desarrolla. Un ejemplo de esto es el conocimiento de los padres o cuidadores sobre la condición clínica del hijo, lo cual conlleva cambios sutiles en el comportamiento de los primeros,

6 EI TEA está probablemente causado por múltiples genes de efecto limitado y el SW es causado por una deleción heterocigótica de algunos de los 28 genes continuos en el cromosoma 7q11.23. (Karmiloff-Smith et al., 2012). 
modificando la interacción diádica. Precisamente Karmiloff-Smith et al. (2012) presentan dos ejemplos para este argumento. Primero, que, a diferencia de los padres de hijos con desarrollo típico, los padres de hijos con síndromes genéticos frecuentemente no les permiten explorar el entorno con igual libertad por medio del chupeteo y gateo, probablemente por un temor ante accidentes, pero implicando una exploración ambiental menos rica. $Y$ segundo, según John \& Mervis (2010), los padres de niños pequeños con $\mathrm{SD}$ les corrigen inmediatamente y vetan las sobre-generalizaciones cuando ellos nombran objetos. Esto se debe a que temen que el niño con menor inteligencia no pueda entender el término correcto si se le permite sobre-generalizar, siendo que para el niño con desarrollo típico esto estimula la formación de categorías dentro del aprendizaje de vocabulario (p.e., al referirse a animales diferentes como gato, el niño empieza a crear una categoría implícita de animales). Conociendo que la categorización es un proceso comprometido o disminuido en varios desordenes del neurodesarrollo, Karmiloff-Smith et al. (2012) proponen que este tipo de suposiciones, sobre lo que un niño con desarrollo atípico puede aprender o no, pueden llevar a los padres a ofrecerles menor variación en los estímulos lingüísticos y, en general, un ambiente menos variado para explorar. ${ }^{7}$

Estas consideraciones sobre la interrelación entre variables genéticas, neurobiológicas y socio-ambientales tienen implicaciones en líneas contemporáneas de investigación sobre la empatía y comprensión de emociones en diferentes trastornos del desarrollo, como el TEA (López \& Rivas, 2014), o sobre las trayectorias de desarrollo entendidas como procesos a lo largo del ciclo vital. Un ejemplo integrativo de estos dos puntos es el estudio de Bennett et al. (2013), el cual reporta un seguimiento prospectivo de niños con TEA desde los 6-8 años hasta los 10-12 años $(\mathrm{Cl}>70)$, con evaluaciones de las habilidades lingüísticas, la solución de problemas no verbales, teoría de la mente (TM) y comportamientos sociales adaptativos. Al realizar análisis de correlación bivariada y de regresión múltiple, encontraron que los resultados en prueba de TM median una asociación con los resultados en pruebas de habilidades lingüísticas, pero no con los resultados en prueba de comportamientos sociales adaptativos. Esto, según los autores, sugiere que un dominio puede constreñir o facilitar otro, en este caso el dominio comunicativo facilita la comprensión de emociones de otros, pero no la adaptación social, apoyando las hipótesis del neuroconstructivismo y, además, plantea que la evaluación de la TM no sería suficiente para indagar los déficits sociales del TEA. Adicionalmente, la revisión de Rinaldi \& Karmiloff-Smith (2017) expone múltiples argumentos y evidencias que sugieren que la evaluación de la inteligencia, que tradicionalmente ha sido una operacionalización simple y transversal, puede extenderse a un análisis del constructo como proceso psicológico dinámico que varía a lo largo del ciclo vital. Esta variabilidad longitudinal e individual se daría dependiendo de

7 De esta forma, los aportes de Karmiloff-Smith también dan cabida a la influencia que los padres ejercen sobre sus hijos como otra dinámica social que contribuye a las variabilidades en el desarrollo cognitivo de acuerdo al estilo educativo parental y a las prácticas parentales específicas con las que se manifiesta su estilo educativo y otorgamiento de cuidados (Darling \& Steinberg, 1993; Jorge \& González, 2017). 
las interacciones fluctuantes entre niveles ontogenéticos y el desarrollo probabilístico de cada dominio cognitivo (general y específico) en cada persona.

Resumiendo las evidencias y posturas expuestas anteriormente, la perspectiva de Karmiloff-Smith et al. (2012) y del neuroconstructivismo plantean la posibilidad de estudiar los efectos de distintos factores de variabilidad de la interacción social sobre el desarrollo cognitivo y cerebral atípico, teniendo en cuenta también las amplias diferencias individuales (cognitivas y sociales) que presentan los pacientes con desórdenes del neurodesarrollo. De esta forma, se sugiere que este tipo de trabajos presentarán nuevas evidencias y puntos de discusión sobre las características del desarrollo humano y la variabilidad de la cognición y la inteligencia en las trayectorias de desarrollo individual.

Finalmente, una perspectiva similar en relación a la interacción social es sugerida de forma independiente por Frisch (2014) y Spunt \& Adolphs (2017), quienes argumentan que la consideración de los conceptos de modularidad y especificidad de dominio debe ser diferentes cuando se investiga el desarrollo de procesos socio-cognitivos. Una razón para esto es que múltiples procesos cognoscitivos posibilitan la adquisición de conocimientos sociales (p.e. el sentido de agencia es posibilitado por procesos de memoria declarativa, percepción y lenguaje). Otro argumento es que la especificidad de dominio puede emerger cuando un módulo que recibe mucha información (a través de múltiples transductores sensoriales o fuentes ambientales, con modulación top-down) está en capacidad de filtrar dicha información para presentar características de un dominio general. En consecuencia, las definiciones de modularidad y especificidad de dominio resultan ambiguas cuando se analiza la forma en que los procesos cognitivos específicos informan a los conocimientos de tipo social y la forma en que los eventos sociales configuran las características de los procesos cognoscitivos. En general, de acuerdo a Spunt \& Adolphs (2017), los estudios en el área de la neurociencia social continuarán aportando a la comprensión de la especificidad de dominio inmersa en contextos sociales dinámicos e informando sobre qué aspectos de la especificidad de dominio pueden constituir el centro del desarrollo típico y atípico de la cognición humana.

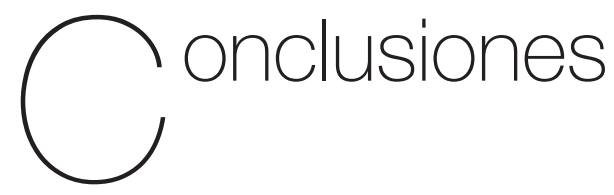

Aunque en este escrito se han abordado brevemente los aportes teóricos e investigativos principales de la autora, vale la pena comentar que su obra fue extensa, con varios centenares de producciones contando libros, capítulos de libros, artículos empíricos y revisiones. La mayor parte de su bibliografía fue escrita con otros investigadores, un hecho que resalta su capacidad de cooperación en la investigación, su interés interdisciplinario y probablemente da cuenta del alcance de su aporte dentro de la psicología y la ciencia cognitiva. Entre otros de sus libros están: "Neurodevelopmental disorders across the lifespan: A neuroconstructivist approach" (Farran \& Karmiloff-Smith, 2012), "Pathways to Language: From foetus to adolescent" (Karmiloff \& Karmiloff-Smith, 2001) y "Baby It's You: A unique insight into the first three years of the developing baby" (Karmiloff-Smith, 
1994), los cuales tuvieron también amplia difusión, siendo el último un libro de divulgación científica.

En conclusión, Annette KarmiloffSmith fue una científica británica y psicóloga del desarrollo que aportó un marco teórico entre la postura constructivista piagetiana y el innatismo de Chomsky y Fodor. Fue una fuerte crítica de los conceptos de modularidad propuestos para la descripción del desarrollo psicológico y propuso fundamentos teóricos y metodológicos integradores para investigar y entender la variabilidad del desarrollo típico y atípico.

Su trabajo posesionó en la comunidad académica una comprensión del desarrollo que supera las posturas dicotómicas y que supone un camino de descripción y explicación de los procesos de cambio que considera e integra los continuos avances en el campo de la neurociencia, la comprensión creciente de los procesos cognitivos en los que inciden las constricciones filogéneticas y las dinámicas ontogenéticas, sin desconocer la incidencia clave del contexto socio-cultural y la interacción no lineal de todos estos factores en el tiempo del desarrollo.

Por todo esto, los aportes de Karmiloff-Smith constituyen un antecedente investigativo que tiene un alto valor e impacto en la psicología al haber planteado líneas de trabajo integrativas entre diferentes ciencias (neurociencia, genética, ciencia cognitiva) para mejorar la capacidad explicativa de la variabilidad compleja que caracteriza el desarrollo cognitivo humano. La obra de Karmiloff-Smith constituye un inspirador ejemplo para investigaciones futuras en psicología que busquen colaboraciones específicas con otras disciplinas y permitan continuar obteniendo evidencias sobre las relaciones entre variables biológicas, psicológicas y sociales que dan forma al desarrollo.

\section{Agradecimientos}

Los autores agradecen a Alejandro Bejarano por la lectura realizada al artículo y sus aportes a la argumentación del mismo.

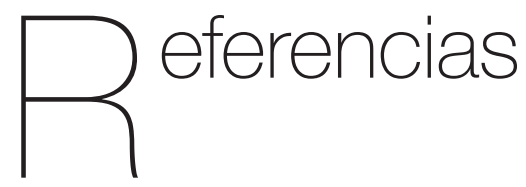

Annaz, D., Karmiloff-Smith, A., Johnson, M.H., \& Thomas, M.S.C. (2009). A crosssyndrome study of the development of holistic face recognition in children with autism, Down syndrome, and Williams syndrome. Journal of Experimental Child Psychology, 102, 456-486. Retrieved from https://doi.org/10.1016/j.jecp.2008.11.005

Association for Psychological Science (2015). Inside the Psychologist's Studio: Annette Karmiloff-Smith. (BJ Casey, Entrevistadora) [Archivo de video]. Retrieved from http://www.psychologicalscience.org/ video/karmiloff-smith-talks-futureof-developmental-science.html\#. WFIZ32CV6Uk

Association for Psychological Science (2016, Diciembre, 20). In Memoriam: Annette Karmiloff-Smith. Washington, D.C.: Association for Psychological Science. Retrieved from https://www. psychologicalscience.org/publications/ observer/obsonline/annette-karmiloffsmith-past-aps-board-member-and- 
leading-expert-in-neurocognitivedevelopment.html

Bennett, T. A., Szatmari, P., Bryson, S., Duku, E., Vaccarella, L., \& Tuff, L. (2013). Theory of mind, language and adaptive functioning in ASD: A neuroconstructivist perspective. Journal of the Canadian Academy of Child and Adolescent Psychiatry, 22(1), 13-19. Retrieved from https://www.ncbi.nlm.nih. gov/pubmed/23390428

Besle, J., Fischer, C., Bidet-Caulet, A., Lecaignard, F., Bertrand, O., \& Giard, M.H. (2008). Visual activation and audiovisual interactions in the auditory cortex during speech perception: Intracranial recordings in humans. The Journal of Neuroscience, 28, 14301-14310. Recuperado de https://doi. org/10.1523/JNEUROSCI.2875-08.2008

Bishop, D.V.M. (2011). What Causes Specific Language Impairment in Children?. Current Directions in Psychological Science, 15(5), 217-221. doi:10.1111/j.1467-8721.2006.00439.x.

Campbell, L.E., Daly, E., Toal, F., Stevens, A., Azuma, R., Karmiloff-Smith, A., etal. (2009a). Brain structural differences associated with the behavioural phenotype in children with Williams syndrome. Brain Research, 1258, 96-107. Retrieved from https://doi. org/10.1016/j.brainres.2008.11.101

Campbell, L.E., Stevens, A., Daly, E., Toal, F., Azuma, R., Karmiloff-Smith, A., et al. (2009b). A comparative study of cognition and brain anatomy between two neurodevelopmental disorders: 22q11.2 deletion syndrome and Williams syndrome. Neuropsychologia, 47(4), 1034-1044. doi: 10.1016/j.neuropsychologia.2008.10.029
Campos, R. (2017). If you want to get ahead, get a good master. Annette KarmiloffSmith: the developmental perspective / Si quieres avanzar, ten una buena maestra. Annette Karmiloff-Smith: la mirada desde el desarrollo. Infancia y Aprendizaje, 41(1), 90137. doi: 10.1080/02103702.2017.1401318

Choudhury, N. \& Benasich, A. (2011). Maturation of auditory evoked potentials from 6-48 months: Prediction to 3 and 4 years language and cognitive abilities. Clinical Neurophysiology, 122, 320-338. Retrieved from https://doi.org/10.1016/j. clinph.2010.05.035

Darling, N., \& Steinberg, L. (1993). Parenting style as context: An integrative model. Psychological Bulletin, 113(3), 487-496. doi: 10.1037/0033-2909.113.3.487

D'Souza, D. \& Karmiloff-Smith, A. (2011). When modularization fails to occur: $A$ developmental perspective. Cognitive Neuropsychology, 28(3), 276-287. doi: 10.1080/02643294.2011.614939

D'Souza, D., D'Souza, H \& KarmiloffSmith, A. (2017). Precursors to language development in typically and atypically infants and toddlers: The importance of embracing complexity. Journal of Child Language, 44, 591-627. doi: 10.1017/ S030500091700006X

Dimitriou, D., Leonard, H.C., Karmiloff-Smith, A., Johnson, M.H., \& Thomas, M.S.C. (2015). Atypical development of configural face recognition in children with autism, Down syndrome, and Williams syndrome. Journal of Intellectual Disability Research, 59(5), 422-438. doi: 10.1111/jir.12141

Elman, J. \& Tyler, L. (2017). Annette KarmiloffSmith obituary: Neuroscientist who 
provided fresh insights into our development as individuals. United Kingdom: The Guardian. Retrieved from https://www. theguardian.com/science/2017/jan/26/ annette-karmiloff-smith-obituary

Elman, J.L., Bates, E., Johnson, M.H., KarmiloffSmith, A., Parisi, D. \& Plunkett, K. (1996). Rethinking Innateness: A Connectionist Perspective on Development. Cambridge, MA: MIT Press.

Farina, M. (2016). Three Approaches to Human Cognitive Development: Neo-Nativism, Neuroconstructivism, and Dynamic Enskilment. The British Journal for the Philosophy of Science, 67(2), 617-641. doi:10.1093/bjps/axu026

Farran, E. \& Karmiloff-Smith, A. (Eds.) (2012). Neurodevelopmental disorders across the lifespan: A neuroconstructivist approach. Oxford: Oxford University Press.

Fodor, J. A. (1983). The Modularity of Mind. Cambridge: MIT Press.

Frisch, S. (2014). How cognitive neuroscience could be more biological-and what it might learn from clinical neuropsychology. Frontiers in Human Neuroscience, 8(541), 1-13. doi: 10.3389/fnhum.2014.00541

Gauthier, I., Skudlarski, P., Gore, J.C., \& Anderson, A.W. (2000). Expertise for cars and birds recruits brain areas involved in face recognition. Nature Neuroscience, 3, 191-197. doi:10.1038/72140

Gottlieb, G. (2007). Probabilistic epigenesis. Developmental Science, 10(1), 1-11. doi: 10.1111/j.1467-7687.2007.00556.x

Gray, V., Karmiloff-Smith, A., Funnell, E., \& Tassabehii, M. (2006). In-depth analysis of spatial cognition in Williams syndrome: A critical assessment of the role of the LIMK1 gene. Neuropsychologia, 44, 679-685. doi: 10.1016/j.neuropsychologia.2005.08.007

John,A.E., \&Mervis, C.B.(2010).Comprehension of the communicative intent behind pointing and gazing gestures by young children with Williams syndrome or Down syndrome. Journal of Speech, Language, and Hearing Research, 53(4), 950-960. doi:10.1044/1092-4388(2009/08-0234)

Johnson, M. H. (2005). Subcortical face processing. Nature Reviews Neuroscience, 6, 766-773. doi: 10.1038/nrn1766

Jorge, E. \& González, C. (2017). Estilos de crianza parental: una revisión teórica. Informes Psicológicos, 17(2), 39-66. doi: 10.18566/infpsic.v17n2a02

Karmiloff, K. \& Karmiloff-Smith, A. (2001). Pathways to language: From foetus to adolescent. Cambridge, MA: Harvard University Press.

Karmiloff-Smith, A. (1979). A Functional Approach to Child Language: A Study of Determiners and Reference. Cambridge: Cambridge University Press.

Karmiloff-Smith, A. (1985). Language and cognitive processes from a developmental perspective. Language and Cognitive Processes, 1(1), 61-85.

Karmiloff-Smith, A. (1992). Beyond Modularity: A Developmental Perspective on Cognitive Science. Cambridge, MA: MIT Press/ Bradford Books.

Karmiloff-Smith, A. (1994). Baby It's You: A unique insight into the first three years of 
the developing baby. London: Ebury Press, Random House.

Karmiloff-Smith, A. (1998a). Development itself is the key to understanding developmental disorders. Trends in Cognitive Sciences, 2(10), 389-398. doi: 10.1016/ S1364-6613(98)01230-3

Karmiloff-Smith, A. (1998b). Is typical development necessarily a window on the normal mind/brain? The case of Williams syndrome. Developmental Science, 1, 273277. doi: 10.1111/1467-7687.00042

Karmiloff-Smith, A., D'Souza, D., Dekker, T.M., Van Herwegen, J., Xu, F., \& Rodic, M. (2012). Genetic and environmental vulnerabilities in children with neurodevelopmental disorders. Procedures of the National Academy of Sciences, 109(Suppl. 2), 1726117265. doi: 10.1073/pnas.1121087109

Köhler, R. (2008). Jean Piaget. New York: Bloomsbury Academic.

Kuhl, P. (2004). Early Language Acquisition: Cracking the Speech Code. Nature Reviews Neuroscience, 5, 831-843. doi:10.1038/nrn1533

Liégeois, F., Connelly, Al., Baldeweg, T., \& Vargha-Khadem, F. (2008). Speaking with a single cerebral hemisphere: fMRI language organization after hemispherectomy in childhood. Brain and Language, 106(3), 195-203. doi: 10.1016/j.bandl.2008.01.010

López, S. \& Rivas, R. (2014). El trastorno del espectro del autismo: Retos, oportunidades y necesidades. Informes Psicológicos, 14(2), 13-31. Recuperado de https://revistas.upb.edu.co/index.php/ informespsicologicos/article/view/5485
Mareschal, D., Johnson, M.K., Sirois, S., Spratling, M.W., Thomas, M.S.C., \& Westermann, G. (2007). Neuroconstructivism: How the Brain Constructs Cognition (Volume 1). Oxford: Oxford University Press.

Marr, D. (1982). Vision. San Francisco, CA: W. H. Freeman.

Moyle, J., Stokes, S.F., \& Klee, T. (2011). Early Language Delay and Specific Language Impairment. Developmental Disabilites Research Reviews, 17, 160-169. doi: 10.1002/ddrr.1110

Pekkola, J., Ojanen, V., Autti, T., Jäskeläinen, I. P., Mottonen, R., Tarkiainen, A., et al. (2005). Primary auditory cortex activation by visual speech: An fMRI study at 3 Tesla. Neuroreport, 16, 125-128. Recuperado de https://www.ncbi.nlm.nih.gov/ pubmed/15671860

Piaget, J. (1983). El lenguaje y el pensamiento en el niño: Estudio sobre la lógica del niño (I) (M. Riani, Trad.). Buenos Aires: Guadalupe. (Trabajo original publicado en 1923).

Piaget, J., \& Inhelder, B. (2000). The psychology of the child. $(\mathrm{H}$. Weaver, Trad.). New York: Basic Books (Trabajo original publicado en 1969).

Piaget, J. (1991). Seis Estudios de Psicología. (J. Marfá, Trad.). Barcelona: Editorial Labor. (Trabajo original publicado en 1964).

Ramus, F. (2004). Should neuroconstructivism guide developmental research?. Trends in Cognitive Sciences, 8(3), 100-101. doi: 10.1016/j.tics.2004.01.002

Rinaldi, L., \& Karmiloff-Smith, A. (2017). Intelligence as a Developing Function: 
A Neuroconstructivist Approach. Journal of Intelligence, 5(2), 18-44. Retrieved from https://doi.org/10.3390/ jintelligence5020018

Rogoff, B. (1990). Apprenticeship in thinking, cognitive development in social contexts. Oxford: Oxford University Press.

Rogoff, B. (1998). Cognition as a collaborative process. En W. Damon (Ed.), Handbook of child psychology: Cognition, perception and language, (pp. 679-744). New York: John Wiley.

Rogoff, B. (2003). The cultural nature of human development. New York: Oxford University Press.

Smith, A. Gilchrist, I., Hood, B., Tassabehji, M., \& Karmiloff-Smith, A. (2009). Inefficient search of large-scale space in Williams syndrome: Further insights on the role of LIMK1 deletion in deficits of spatial cognition. Perception, 38(5), 694-701. doi: 10.1068/p6050

Spunt, R.P., \& Adolphs, R. (2017). A new look at domain specificity: Insights from social neuroscience. Nature Reviews Neuroscience, 18, 559-567. doi: 10.1038/ nrn.2017.76

Steele, A., Scerif, G., Cornish, K., \& KarmiloffSmith, A. (2013). Learning to read in Williams syndrome and Down syndrome: syndrome-specific precursors and developmental trajectories. Journal of Child Psychology and Psychiatry, 54(7), 754-762. doi: 10.1111/jcpp.12070

Stein, B. E., \& Stanford, T. R. (2008). Multisensory integration: Current issues from the perspective of the single neuron.
Nature Reviews Neuroscience, 9, 255-266. doi:10.1038/nrn2331

Stevens, A.F., Campbell, L.E., Morris, R, Simonoff, E., Murphy, D.G., KarmiloffSmith, A., et al. (2005). Psychiatric profile of children with velo-cardio-facial syndrome (VCFS). Schizophrenia Bulletin, 31(2), 377378. Retrieved from https://doi.org/10.1093/ schbul/sbiO24

Stiles, J., Bates, E., Thal, D., Trauner, D., \& Reilly, J. (2002). Linguistic and spatial cognitive development in children with preand perinatal focal brain injury: A ten-year overview from the San Diego Longitudinal Project. En M.H. Johnson, Y. Munakata, \& R. Gilmore. (Eds.), Brain development and cognition: A reader (pp. 643-664). Oxford, UK: Blackwell.

Thomas, M. (2017). Annette Karmiloff-Smith 1938-2016: An appreciation from Professor Michael Thomas. The Psychologist, 30, 9. Retrieved from https://thepsychologist. bps.org.uk/volume-30/february-2017/ annette-karmiloff-smith-1938-2016

Tolchinsky, L. (1996). Más allá de la modularidad de Annette Karmiloff Smith o cómo hacer de la psicología del desarrollo una ciencia relevante. Anuario de Psicología, 69, 199211. Recuperado de http://revistes.ub.edu/ index.php/Anuario-psicologia/article/ view/9098/11612

Tolchinsky, L. (2017). Going beyond. In tribute to Annette Karmiloff-Smith. Infancia $Y$ Aprendizaje - Journal for the Study of Education and Development, 41(1), 56-83. doi: 10.1080/02.2017.1402506.

Van de Riet, W.A.C., Grezes, J., \& de Gelder, B. (2009). Specific and common brain regions involved in the perception of 
faces and bodies and the representation of their emotional expressions. Social Neuroscience, 4(2), 101-120. doi: 10.1080/17470910701865367

Van Herwegen, J., Ansari, D., Xu, F., \& Karmiloff-Smith, A. (2008). Small and large number processing in infants and toddlers with Williams syndrome. Developmental Science, 11(5), 637-643. doi: 10.1111/j.1467-7687.2008.00711.x

Werker, J.F. \& Polka, L. (1993). Developmental changes in speech perception: New challenges and new directions. Journal of Phonetics, 21, 83-101.

Westermann, G., Mareschal, D., Johnson, M.K., Sirois, S., Spratling, M.W., \& Thomas, M.S.C. (2007). Neuroconstructivism. Developmental Science, 10(1), 75-83. doi: 10.1111/j.1467-7687.2007.00567.x

Wiseman, F.K., Al-Janabi, T., Hardy, J., Karmiloff-Smith, A., Nizetic, D., Tybulewicz, V.L.J., et al. (2015). A genetic cause of Alzheimer disease: mechanistic insights from Down syndrome. Nature Reviews Neuroscience, 16(9), 564-574. doi:10.1038/ nrn3983 